\title{
Probabilistic Enhancement of Classical Robustness Margins: The Unirectangularity Concept ${ }^{\dagger}$
}

\author{
C. M. Lagoa \\ University of Wisconsin \\ Madison, WI 53706 \\ lagoa@cae.wisc.edu
}

\author{
P. S. Shcherbakov \\ Moscow Institute of Control Sciences \\ Moscow, Russia \\ sherba@ipu.rssi.ru
}

\author{
B. R. Barmish \\ University of Wisconsin \\ Madison, WI 53706 \\ barmish@engr.wisc.edu
}

\begin{abstract}
The focal point of this paper is a control system subjected to parametric uncertainty. Motivated by the newly emerging theory of probabilistic robustness, the risk of performance violation is assessed with uncertainty bounds which exceed classical deterministic margins. For a wide class of problems, the Uniformity Principle (UP) makes it possible to estimate the probability of performance satisfaction with almost no apriori statistical information about the uncertainty. The application of the UP is, however, limited to problems satisfying certain convexity and symmetricity conditions. This paper extends the application of the UP by working with a so-called unirectangularity condition.
\end{abstract}

\section{Introduction}

The takeoff point for this paper is a control system described in terms of an $\ell$-tuple of uncertain parameters $q=\left(q_{1}, q_{2}, \ldots, q_{\ell}\right)$. Each $q \in \mathbf{R}^{\ell}$ defines a system $\mathcal{S}_{q}$ and $Q \subset \mathbf{R}^{\ell}$ is a given hyper-rectangle defining the shape for the uncertainty. Within this standard setting, classical robustness margin problems are formulated by allowing the radius of the uncertainty bounding set $r \geq 0$ to be variable. That is, with

$$
Q_{r} \doteq r Q,
$$

we obtain a family of systems indexed by $q \in Q_{r}$. Let $\mathcal{P}$ denote a desired property to be satisfied by every system in the family; e.g., Property $\mathcal{P}$ might represent a specification involving stability, overshoot or rise time. Classical robustness theory is aimed at checking if all members of the uncertain family possesses Property $\mathcal{P}$ and determining the classical robustness margin

$$
r_{\max } \doteq \sup \left\{r: \mathcal{S}_{q} \text { has Property } \mathcal{P} \text { for all } q \in Q_{r}\right\} .
$$

For many problems of practical interest, however, the quantity $r_{\max }$ is difficult to compute. Instead, some algorithm $\mathcal{A}$ is applied to obtain a provable robustness radius $r_{0} \leq r_{\max }$.

In this paper, motivated by [1]-[5], we adopt the

${ }^{\dagger}$ Funding for this research was provided by the National Science Foundation under Grant ECS-9418709. probabilistic point of view and assume that $q$ is a random vector having support $Q_{r}$. In order to quantify conservatism of deterministic robustness margins, a natural question is: How far can the uncertainty radius $r$ be extended beyond the classical margin $r_{0}$ in exchange of a small risk probability $p=\varepsilon$ that Property $\mathcal{P}$ is violated? For risk $\varepsilon>0$, this leads to the notion of a risk-adjusted robustness margin $R_{0}(\varepsilon)$ as in [6]. Since it is natural to evaluate $R_{\max }(\varepsilon)$ via Monte Carlo sampling, the following question arises: What probability distribution should be assumed for $q$ ? Since such an apriori probability distribution for $q$ is typically unavailable in a robustness setting, this difficulty is circumvented via the new framework in [5]; i.e., we consider distributional robustness. In this new setting, a class of probability density functions $\mathcal{F}_{r}$ is specified for $q$ and we define

$$
\begin{aligned}
R_{\max }(\varepsilon) \doteq \sup \{r \geq 0: \\
\left.\quad \operatorname{Prob}\{\mathcal{P} \text { holds }\} \geq 1-\varepsilon \text { for all } f \in \mathcal{F}_{r}\right\} .
\end{aligned}
$$

Associated with the determination of $R_{\max }(\varepsilon)$ above is the target set or the set of good parameters

$$
Q_{\text {good }} \doteq\left\{q \in \mathbf{R}^{\ell}: \text { Property } \mathcal{P} \text { is satisfied by } \mathcal{S}_{q}\right\} \text {. }
$$

Hence, the $R_{\max }(\varepsilon)$ formula above is equivalent to

$$
\begin{aligned}
R_{\max }(\varepsilon) \doteq \sup \{r \geq 0: & \\
\quad & \left.\operatorname{Prob}\left\{q^{f} \in Q_{\text {good }}\right\} \geq 1-\varepsilon \text { for all } f \in \mathcal{F}_{r}\right\}
\end{aligned}
$$

where $q^{f}$ is the random variable with probability density function $f \in \mathcal{F}_{r}$.

The definition of the class of probability density functions $\mathcal{F}_{r}$ is motivated in large measure by manufacturing considerations: If a system parameter includes uncertainty $q_{i}$ with known bounds $\left|q_{i}\right| \leq r_{i}$, then the use of the paradigm in [5] only requires minimal apriori information about the density function $f_{i}\left(x_{i}\right)$ for $q_{i}$. Namely, $q_{i}$ has zero mean, $f_{i}\left(x_{i}\right)$ is symmetric about $x_{i}=0$ and large manufacturing deviations are less probable than small ones. That is, if $0 \leq x_{a} \leq x_{b} \leq r_{i}$, then $f_{i}\left(x_{b}\right) \leq f_{i}\left(x_{a}\right)$. This situation is depicted in Figure 1 with $r=20$. 


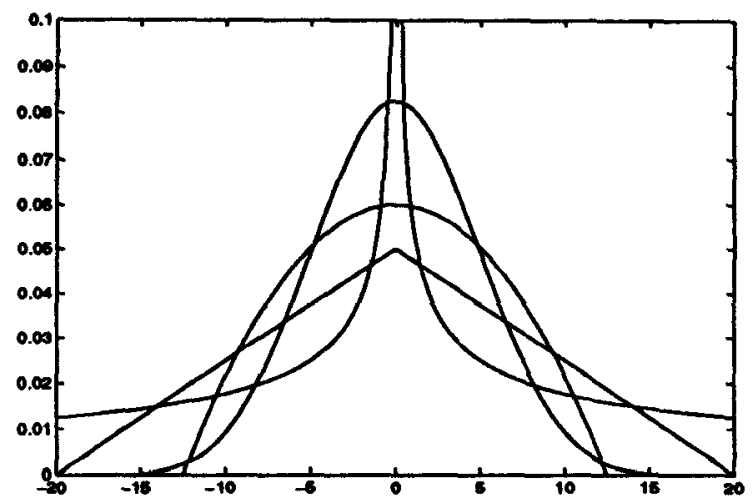

Figure 1: Admissible Distributions for $q_{i}$

As in a traditional robustness setting, only bounds on $q_{i}$ are assumed and no apriori statistics are needed in order to carry out probabilistic robustness analysis. In other words, modulo the mild assumptions above, the results obtained are distribution-free.

With the type of setup above, reference [5] concentrates on the case when the target $Q_{\text {good }}$ is a convex symmetric set. The Uniformity Principle provides a rationale for sampling with the uniform distribution. In other words, for fixed radius of uncertainty $r$, it is proven in [5] that when $\boldsymbol{Q}_{\text {good }}$ is convex and symmetric

$$
\min _{f \in \mathcal{F}_{r}} \operatorname{Prob}\left\{q^{f} \in Q_{g o o d}\right\}=\operatorname{Prob}\left\{q^{u_{r}} \in Q_{g o o d}\right\}
$$

where $q^{u_{r}}$ denotes the random vector with uniform distribution over $Q_{r}$. When the target set is convex but not symmetric, the results in [6] show how the theory in [5] can still be used. This is accomplished via a symmetrization process and illustrated for the case of linear matrix inequalities. A so-called sharp probabilistic estimator $R_{0}(\varepsilon)$ is obtained in lieu of $R_{\max }(\varepsilon)$.

The notion of a sharp probabilistic estimator is also central to this paper. If $r_{0} \leq r_{\max }$ is a robustness radius provided by some algorithm $\mathcal{A}$, we seek a risk-adjusted robustness radius $R_{0}(\varepsilon)$ which is sharp in the sense that

$$
R_{\max }(\varepsilon) \geq R_{0}(\varepsilon) \geq r_{0}
$$

for all risk levels $0 \leq \varepsilon \leq 1$. Notice that, with uncertainty $q$ restricted to the box of radius $R_{0}(\varepsilon)$, it is assured that

$$
\operatorname{Prob}\left\{q^{f} \in Q_{\text {good }}\right\} \geq 1-\varepsilon
$$

for all admissible densities $f \in \mathcal{F}_{R_{0}(\varepsilon)}$. In contrast to [6], however, we make no convexity assumption on $Q_{\text {good }}$.

1.1 The Unirectangularity Concept Instead of working with a convex symmetrization of $Q_{g o o d}$ as in [6], we work with its unirectangular internal hull which is denoted by $Q_{g o o d, u n i}$ (see Section 2 ) and the obvious inequality

$$
\operatorname{Prob}\left\{q \in Q_{\text {good }}\right\} \geq \operatorname{Prob}\left\{q \in Q_{\text {good,uni }}\right\} \geq 1-\varepsilon .
$$

The method to obtain $R_{0}(\varepsilon)$ is applicable to almost all problems for which there exists a deterministic algorithm $\mathcal{A}$ leading to a robustness margin $r_{0}$. The procedure in this paper can be used to amplify $r_{0}$ to obtain a larger risk-adjusted radius $R_{0}(\varepsilon) \geq r_{0}$ based upon sampling uncertainty boxes $Q_{r}$. The key idea involves repeated applications of the algorithm $\mathcal{A}$. For each sample $q^{k}$, the algorithm $\mathcal{A}$ is applied to an appropriately constructed deterministic problem and a "count" is kept on the number of successes (satisfaction of $\mathcal{P}$ ) versus failures. The reliability of the estimate of the probability of performance depends on the number of samples but is easily shown to be independent of the uncertainty dimension.

\section{A Theorem Stated in Probabilistic Terms}

The highlight of this section, Theorem 2.5, is a "stepping stone" for probabilistic robustness analysis provided in Section 3.

2.1 Random Variables: We consider a random vector $X=\left(X_{1}, X_{2}, \ldots, X_{\ell}\right) \in \mathbf{R}^{\ell}$ with components $X_{i}$ which are independent and satisfy the assumptions in [5]. Namely, the support set for each $X_{i}$ is an interval $\left[-r_{i}, r_{i}\right]$. Without loss of generality, we take all $r_{i}=r$ via an appropriate scaling of the $X_{i}$. Next, for each $X_{i}$, an admissible density function $f_{i}$ is required to be symmetric with respect to zero and nonincreasing in $\left|x_{i}\right|$. The class of such densities, whose support is the hypercube defined by $r$, is denoted by $\mathcal{F}_{r}$.

Given any admissible density function $f \in \mathcal{F}_{r}$, let $X^{f}$ to be the associated random vector. The uniform distribution for $X$ is denoted by $u_{r}$ and $u_{r, i}$ represents the uniform distribution for the component $X_{i}$.

2.2 Definition of Unirectangularity: Given any $x \doteq\left(x_{1}, x_{2}, \ldots, x_{\ell}\right)$, its rectangular projection is

$\mathcal{R}(x) \doteq\left\{\left(\alpha_{1} x_{1}, \ldots, \alpha_{\ell} x_{\ell}\right): \alpha_{i} \in[0,1]\right.$ for $\left.i=1, \ldots, \ell\right\}$.

With this notation, a set $\mathcal{X} \subseteq \mathbf{R}^{\ell}$ is said to be unirectangular if $\mathcal{R}(x) \subseteq \mathcal{X}$ for all $x \in \mathcal{X}$.

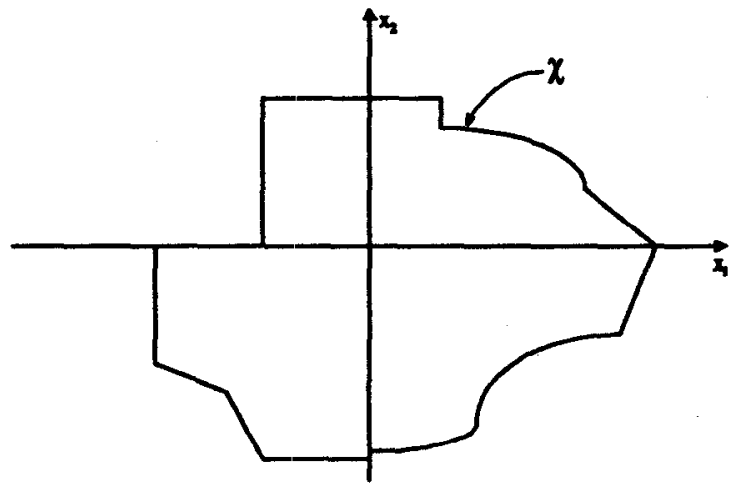

Figure 2: A Unirectangular Set $\mathcal{X}$ in $\mathbf{R}^{2}$

2.3 Example: For $\ell=2$, a unirectangular set is shown in Figure 2. To see that unirectangularity is different 
from convexity, note that an $\ell$-dimensional convex ellipsoid $\mathcal{X}$ is not necessarily unirectangular; e.g., see Figure

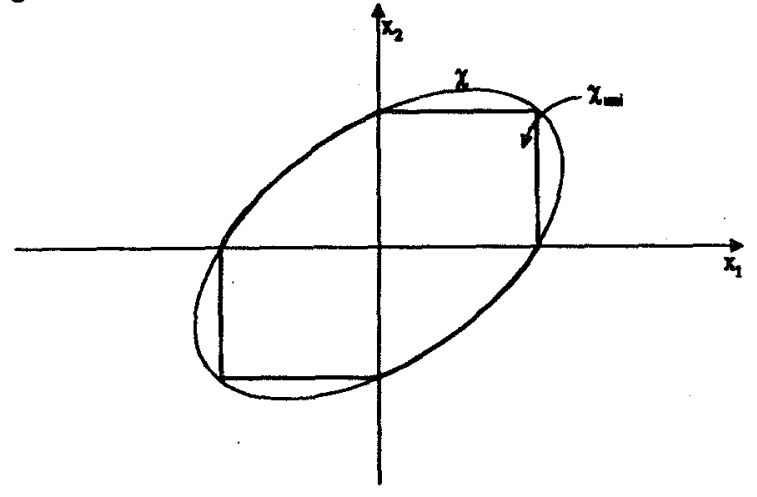

Figure 3: Unirectangular Internal Hull

2.4 Definition of Unirectangular Internal Hull: Given $\mathcal{X} \subseteq \mathbf{R}^{\ell}$, its unirectangular internal hull is defined by

$$
\mathcal{X}_{\text {uni }} \doteq\{x \in \mathcal{X}: \mathcal{R}(x) \subseteq \mathcal{X}\}
$$

This construction is illustrated in Figure 3 with $\mathcal{X}$ being a two-dimensional ellipse.

2.5 Theorem: Let $\mathcal{X} \subseteq \mathbf{R}^{\ell}$ be a unirectangular set; i.e., $\mathcal{X}_{\text {uni }}=\mathcal{X}$. Then, given $r>0$,

$$
\min _{f \in \mathcal{F}_{r}} \operatorname{Prob}\left\{X^{f} \in \mathcal{X}\right\}=\operatorname{Prob}\left\{X^{u_{r}} \in \mathcal{X}\right\}
$$

Proof: We first take $\ell=1$ and make note of a simple property associated with probability density functions $f \in \mathcal{F}_{r}$. Namely, for any $\varepsilon \geq 0$ and $\delta \geq 0$, a straightforward mass balancing argument yields

$$
\min _{f \in \mathcal{F}_{r}} \operatorname{Prob}\left\{X^{f} \in[-\delta, \varepsilon]\right\}=\operatorname{Prob}\left\{X^{u_{r}} \in[-\delta, \varepsilon]\right\} .
$$

Now, let $\ell$ be any positive integer and take $f\left(x_{1}, x_{2}, \ldots, x_{\ell}\right) \doteq f_{1}\left(x_{1}\right) f_{2}\left(x_{2}\right) \cdots f_{\ell}\left(x_{\ell}\right)$ to be any density function in $\mathcal{F}_{r}$. Letting $g\left(x_{1}, x_{2}, \ldots, x_{\ell}\right) \doteq$ $u_{r, 1}\left(x_{1}\right) f_{2}\left(x_{2}\right) \cdots f_{\ell}\left(x_{\ell}\right)$, it is noted that $g \in \mathcal{F}_{r}$, we claim that $\operatorname{Prob}\left\{X^{f} \in \mathcal{X}\right\} \geq \operatorname{Prob}\left\{X^{g} \in \mathcal{X}\right\}$. To prove this claim, note that

$$
\begin{aligned}
& \operatorname{Prob}\left\{X^{f} \in \mathcal{X}\right\}-\operatorname{Prob}\left\{X^{g} \in \mathcal{X}\right\} \\
&=\int_{\mathcal{X}^{\ell-1}} f_{2}\left(x_{2}\right) \cdots f_{n}\left(x_{\ell}\right) \\
&\left(\int_{\mathcal{X}^{1}\left(x_{2}, \ldots, x_{\ell}\right)} f_{1}\left(x_{1}\right)-u_{r, 1}\left(x_{1}\right) d x_{1}\right) d x_{2} \cdots d x_{\ell}
\end{aligned}
$$

where

$$
\begin{aligned}
\mathcal{X}^{\ell-1} \doteq\left\{\left(x_{2}, \ldots, x_{\ell}\right) \in \mathbf{R}^{\ell-1}:\right. \\
\left.\left(x_{1}, x_{2}, \ldots, x_{\ell}\right) \in \mathcal{X} \text { for some } x_{1} \in \mathbf{R}\right\}
\end{aligned}
$$

and $\mathcal{X}^{\mathbf{1}}\left(x_{2}, \ldots, x_{\ell}\right) \doteq\left\{x_{1} \in \mathbf{R}:\left(x_{1}, x_{2}, \ldots, x_{\ell}\right) \in \mathcal{X}\right\}$ Since $\mathcal{X}$ is unirectangular, the interval $\mathcal{X}^{1}\left(x_{2}, \ldots, x_{\ell}\right)$ contains zero. Applying the result for $\ell=1$,

$$
\int_{\mathcal{X}^{1}\left(x_{2}, \ldots, x_{\ell}\right)} f_{1}\left(x_{1}\right)-u_{r, 1}\left(x_{1}\right) d x_{1} \geq 0
$$

leads to inequality $\operatorname{Prob}\left\{X^{f} \in \mathcal{X}\right\} \geq \operatorname{Prob}\left\{X^{g} \in \mathcal{X}\right\}$. Now repeating the reasoning above for the remaining $\ell-1$ coordinates, for $f \in \mathcal{F}_{r}$, we arrive at the inequality $\operatorname{Prob}\left\{X^{f} \in \mathcal{X}\right\} \geq \operatorname{Prob}\left\{X^{u_{r}} \in \mathcal{X}\right\}$.

\section{Sharp Estimator}

For each $f \in \mathcal{F}_{r}$, the associated random vector is denoted by $q^{f}$. The numerical method to be used is based upon the theorem below.

3.1 Theorem: Suppose $0<r_{0} \leq r_{\max }$ is a classical robustness margin and $Q_{*} \subset \mathbf{R}^{\ell}$ is a unirectangular set such that $Q_{r_{0}} \subseteq Q_{*} \subseteq Q_{\text {good }}$. Then, the quantity

$$
R_{0}(\varepsilon) \doteq \sup \left\{r: \min _{f \in \mathcal{F}_{r}} \operatorname{Prob}\left\{q^{f} \in Q_{*}\right\} \geq 1-\varepsilon\right\}
$$

is a sharp probabilistic estimator in the sense defined in Section 1 ; i.e., $R_{\max }(\varepsilon) \geq R_{0}(\varepsilon) \geq r_{0}$ for all $0 \leq \varepsilon \leq 1$. Moreover, for any $r>0$,

$$
\min _{f \in \mathcal{F}_{r}} \operatorname{Prob}\left\{q^{f} \in Q_{*}\right\}=\operatorname{Prob}\left\{q^{u_{r}} \in Q_{*}\right\}
$$

Proof: First, given any $0 \leq \varepsilon \leq 1$, the inequality $R_{\max }(\varepsilon) \geq R_{0}(\varepsilon)$ is a straightforward consequence of the definitions in Section 1. Next, given any $r \leq r_{0}$, the set containments $Q_{r} \subseteq Q_{r_{0}} \subseteq Q_{\text {good }}$ and $Q_{r} \subseteq Q_{*}$ guarantees that for any $f \in \mathcal{F}_{r}$ and $r \leq r_{0}$, we have Prob $\left\{q^{f} \in Q_{*}\right\}=1$. Hence, for any $0 \leq \varepsilon \leq 1$, the inequality $R_{0}(\varepsilon) \geq r_{0}$ holds and it follows that $R_{0}(\varepsilon)$ is a sharp probabilistic estimator. Next, note that $Q_{*}$ is a unirectangular set. By Theorem 2.5, the minimum probability that $q^{f} \in Q_{*}$ is attained by the uniform distribution $u_{r}$.

3.2 Remark: In the numerical method to follow, the set $Q_{*}$ is obtained implicitly. For the case when the algorithm $\mathcal{A}$ is exact in the sense that $\mathcal{A}\left(Q_{r}\right)=1$ if and only if Property $\mathcal{P}$ is satisfied for all $q \in Q_{r}$, the estimate of $R_{0}(\varepsilon)$ corresponds to the case $Q_{*}=Q_{\text {good,uni }}$.

3.3 The Deterministic Algorithm: Consider an algorithm $\mathcal{A}$ which is used to compute a robustness margin $r_{0} \leq r_{\max }$. Given any uncertainty box $Q_{r}$, let

$\mathcal{A}\left(Q_{r}\right) \doteq \begin{cases}1 & \text { if Property } \mathcal{P} \text { holds for all } q \in Q_{r} \\ 0 & \text { otherwise. }\end{cases}$

For example, if $\mathcal{A}$ corresponds to testing some inequality (a sufficient condition) guaranteeing Property $\mathcal{P}$, then $\mathcal{A}\left(Q_{r}\right)=1$ indicates that the inequality is satisfied for all $q \in Q_{r}$. Hence,

$$
r_{\max } \geq r_{0}=\sup \left\{r: \mathcal{A}\left(Q_{r}\right)=1\right\}
$$


3.4 Construction of a Sharp Estimator: Let $N$ denote the number of samples of $q$ to be taken and let $\left[0, r^{+}\right]$be the interval for radius $r$ to be considered. For $r \in\left[0, r^{+}\right]$, the objective is to generate a unirectangular set $Q_{*}$ satisfying $Q_{r_{0}} \subseteq Q_{*} \subseteq Q_{\text {good, uni }}$ and an associated estimate $\hat{\mathrm{p}}_{\boldsymbol{r}}$ of the quantity

$$
p_{r} \doteq \operatorname{Prob}\left\{q^{u_{r}} \in Q_{*}\right\}
$$

This is accomplished by drawing samples $q^{1}, q^{2}, \ldots, q^{N}$ uniformly distributed over $Q_{r}$. For each sample $q^{k}$, let $Q^{k} \doteq \mathcal{R}\left(q^{k}\right)$ be its rectangular projection and obtain, via repeated application of $\mathcal{A}$, the estimate

$$
\widehat{\mathrm{p}}_{\mathrm{r}} \doteq \frac{\sum_{i=1}^{N} \max \left\{\mathcal{A}\left(Q^{k}\right), I_{Q_{r_{0}}}\left(q^{k}\right)\right\}}{N}
$$

where $I_{Q}(\cdot)$ denotes the indicator function on a set $Q$; i.e., $I_{Q}(q)=1$ if $q \in Q$ and $I_{Q}(q)=0$ otherwise. Finally, an estimate $\widehat{R}_{0}(\varepsilon)$ of $R_{0}(\varepsilon)$ is given by

$$
\widehat{R}_{0}(\varepsilon) \doteq \sup \left\{r \in\left[0, r^{+}\right]: \widehat{\mathrm{p}}_{r} \geq 1-\varepsilon\right\} .
$$

Note that in practice, the sweep of radius $r \in\left[0, r^{+}\right]$ requires discretization.

\section{Numerical Examples}

In this section, we illustrate the preceding theory.

4.1 Interval Polynomial Family: This example involves an interval polynomial family described by

$$
p(s, q)=p_{0}(s)+\sum_{i=1}^{\ell} g_{i} s^{i-1}
$$

with uncertainty bound $q \in Q_{r}$ and nominal polynomial $p_{0}(s)$ of degree $n>\ell-1$. Now, $\mathcal{A}\left(Q^{k}\right)=1$ if and only if the four Kharitonov polynomials are stable. With lightly damped nominal polynomial

$$
p_{0}(s)=(s+1)^{12}(s+0.001+j)(s+0.001-j),
$$

it is first noted that Kharitonov's Theorem leads to $r_{\max } \approx 0.021$ for the deterministic problem.

The computation of $R_{0}(\varepsilon)$ is now illustrated for $\varepsilon=$ $0.00064, r_{+}=0.03 \approx 1.43 r_{0}$ and $N=100,000 \mathrm{sam}-$ ples. Figure 4 shows the plot of $\hat{p}_{r_{+}}$as a function of the number of samples $N$. For $N \geq 15,000$, the accuracy of $\widehat{p}_{r_{+}}$is within $10^{-3}$ of its steady state value, which is approximately $\hat{\mathrm{p}}_{r_{+}} \approx 0.99936$. For $N \geq 70,000, \hat{\mathrm{p}}_{r_{+}}$ appears to be at its steady value. Here, for a risk of $\varepsilon=$ 0.00064 , we obtain $\widehat{R}_{0}(\varepsilon) \approx 0.03$. In other words, if the deterministic robustness margin is exceeded by $43 \%$, an estimated risk of instability of $\varepsilon \approx 0.00064$ is obtained.

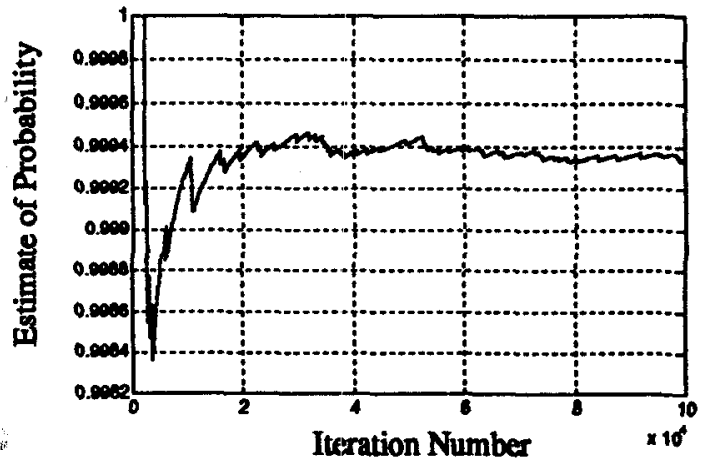

Figure 4: Probability for Example 4.1

4.2 Quadratic Stability: An uncertain system is specified by the state equation $\dot{x}=A x$ with $x \in \mathbf{R}^{n}$ and uncertain matrix is written as $A=A_{0}+\Delta A$ with stable nominal $A_{0}$ and uncertainty $\triangle A$. The entries of $\Delta A$ are assumed to satisfy $\left|\Delta A_{i j}\right| \leq r s_{i j}$ where $s_{i j} \geq 0$ define the shape of the uncertainty set. With $\ell=n^{2}$ and $Q_{r} \subset \mathbf{R}^{\ell}$ denoting the uncertainty bounding set, $P=P^{T}>0$ is taken to be the solution of the classical Lyapunov equation $A_{0}^{T} P+P A_{0}=-I$ for $A_{0}$ and a lower bound $r_{0}$ for the robustness margin

$$
r_{\max }=\sup \left\{r: A^{T} P+P A<0 \text { for all } \Delta A \in Q_{r}\right\}
$$

is used. To this end, the lower bound

$$
r_{0} \doteq \frac{1}{2 \max _{\triangle A \in Q_{1}}\|P \Delta A\|}
$$

is easy to establish for general matrix norms. Since the matrix norm above is a convex function with respect to the entries of $\Delta A$, the maximum above need only be taken over extremal combinations $\Delta A_{i j}= \pm s_{i j}$.

To illustrate the theory in this paper, a matrix norm is exploited which makes it possible to avoid the combinatorially explosive vertex sweep above. Namely with matrix norm $\|M\|_{\infty}$ being the largest column sum for $M$, the stability radius above is readily shown to be

$$
r_{0}=\frac{1}{2 \max _{i} \bar{p}_{i}^{T} s}
$$

with $s$ having $i$-th component which is the $i$-th column sum of $S$ and $\bar{p}_{i} \doteq\left[\left|p_{1, i}\right|\left|p_{2, i}\right| \cdots\left|p_{\ell, i}\right|\right]^{T}$ obtained from the ith column of $P$.

Given a sample $\Delta A^{k}$ obtained using the uniform distribution, the rectangular projection $Q^{k}=$ $\mathcal{R}\left(\Delta A^{k}\right) \subseteq \mathbf{R}^{\ell}$ is used as follows: First, the nominal matrix $A_{0, k} \doteq A_{0}+\frac{1}{2} \Delta A^{k}$ and shaping matrix $S^{k} \doteq \frac{1}{2}\left|\Delta A^{k}\right|$ are defined, where $\left|\Delta A^{k}\right|$ is the matrix whose entries are $\left|\Delta A_{i j}^{k}\right|$. Subsequently, $\mathcal{A}\left(Q^{k}\right)=1$ if the computed value of $r_{0}$ exceeds the magnitude of the smallest eigenvalue of $A_{0, k}^{T} P+P A_{0, k}$. With

$$
A_{0}=\left[\begin{array}{rrr}
-2 & -2 & 0 \\
1 & 0 & 0 \\
1 & 0 & -2
\end{array}\right]
$$


and randomly generated shaping matrix

$$
S=\left[\begin{array}{lll}
0.1651 & 0.9394 & 0.5691 \\
0.2451 & 0.4727 & 0.1457 \\
0.7004 & 0.4014 & 0.3141
\end{array}\right],
$$

the estimate $r_{0} \approx 0.18$ was obtained. The computation of $\widehat{R}_{0}(\varepsilon)$ was carried out for $\varepsilon=0.0044, r^{+}=2.6 r_{0}$ and $N=200,000$. This yielded $\widehat{R}_{0}(\varepsilon) \approx 2.6 r_{0}$, which is a $260 \%$ improvement with the estimated risk of instability being $\varepsilon \approx 0.0044$ at most.

4.3 Polytope of Polynomials: A polytope of polynomials described by $p(s, q)=p_{0}(s)+\sum_{i=1}^{\ell} q_{i} p_{i}(s)$ is considered with stable nominal $p_{0}(s)$ of degree $n$, uncertainty polynomials $p_{i}(s)$ with $\operatorname{deg} p_{i}<n$ and $q \in Q_{r}$. According to [8], the family of polynomials is robustly stable if and only if $p_{0}(s)$ is stable and a specially constructed function $\tau(\omega)$ is positive for all frequencies $\omega \in[0, \infty)$. To obtain an estimate of $R_{0}(\varepsilon)$, we take $\ell=14$, nominal

$$
\begin{array}{r}
p_{0}(s)=s^{8}+11.62 s^{7}+46.49 s^{6}+91.51 s^{5}+113.19 s^{4} \\
+108.14 s^{3}+72.52 s^{2}+30.31 s+9.90
\end{array}
$$

and uncertainty polynomials $p_{i}(s)=\sum_{j=0}^{7} a_{i, 8-j} s^{j}$ whose coefficients $a_{i j}$ are randomly generated and correspond to the entries of the matrix

$$
\begin{aligned}
& A= \\
& {\left[\begin{array}{rrrrrrrr}
-0.17 & -0.26 & -0.27 & 0.23 & 0.00 & -0.02 & 0.23 & -0.19 \\
-0.27 & -0.05 & 0.14 & -0.14 & 0.01 & 0.26 & -0.16 & -0.21 \\
0.11 & 0.11 & -0.10 & -0.04 & -0.11 & -0.27 & -0.12 & 0.04 \\
0.11 & 0.05 & 0.08 & 0.16 & 0.29 & 0.16 & -0.09 & 0.18 \\
0.26 & 0.26 & 0.15 & -0.01 & 0.00 & 0.16 & 0.01 & -0.28 \\
-0.07 & 0.21 & 0.29 & -0.16 & -0.14 & 0.20 & 0.05 & 0.02 \\
0.01 & 0.02 & -0.08 & -0.14 & -0.25 & -0.22 & 0.21 & 0.00 \\
0.20 & -0.24 & -0.15 & -0.08 & 0.27 & -0.29 & -0.05 & 0.27 \\
-0.28 & 0.09 & 0.29 & -0.20 & -0.26 & 0.11 & 0.20 & 0.15 \\
-0.27 & -0.05 & 0.13 & -0.01 & 0.00 & 0.22 & -0.14 & 0.03 \\
0.02 & 0.12 & 0.15 & 0.24 & -0.07 & 0.08 & -0.05 & 0.23 \\
0.10 & 0.25 & 0.09 & 0.25 & -0.13 & 0.14 & 0.02 & 0.07 \\
-0.30 & 0.16 & -0.26 & -0.26 & 0.25 & 0.14 & -0.02 & 0.21 \\
-0.07 & -0.14 & 0.08 & 0.24 & 0.02 & 0.30 & -0.13 & -0.20
\end{array}\right] .}
\end{aligned}
$$

Using [8], we obtain $r_{0} \approx r_{\max } \approx 0.742$ and the procedure presented in Section 3.4 is now carried out with $r^{+}=5 r_{0}$ and $N=50,000$. The theory is demonstrated by generating a plot of the estimated amplification function

$$
\widehat{A}(\varepsilon) \doteq \frac{\widehat{R}_{0}(\varepsilon)}{r_{0}} .
$$

in Figure 5. For instance, the size of the uncertainty box can be doubled with risk of instability $\varepsilon \approx 0.0111$.

\section{More General Product Space Results}

The motivation for a generalization of Theorem 2.5 is quite simple: In many problems the uncertain parameters are often decomposable into disjoint groups which each have their own uncertainty description.

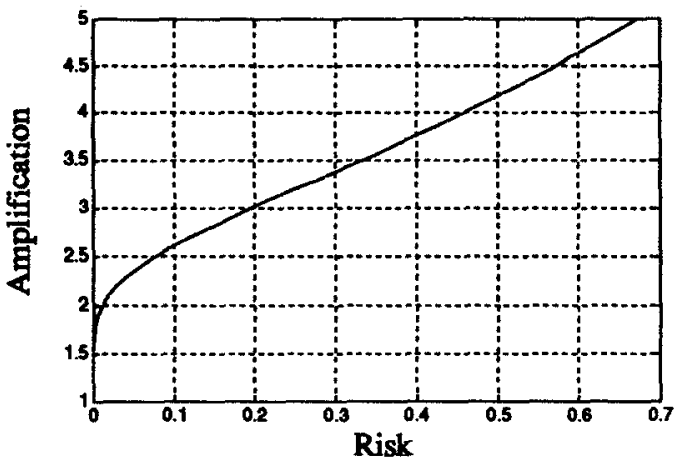

Figure 5. Amplification for Example 4.3

To motivate the result to follow, consider a control system involving two system matrices each undergoing unstructured perturbation; e.g., say $A_{1} \in \mathbf{R}^{n_{1} \times n_{2}}$ and $A_{2} \in \mathbf{R}^{n_{3} \times n_{4}}$ are the nominal matrices and their perturbed versions are

$$
A_{1}+\Delta A_{1} \in \mathbf{R}^{n_{1} \times n_{2}} ; A_{2}+\Delta A_{2} \in \mathbf{R}^{n_{3} \times n_{4}}
$$

with classical singular value bounds

$$
\left\|\Delta A_{1}\right\|=\bar{\sigma}\left(\Delta A_{1}\right) \leq r_{1} ; \quad\left\|\Delta A_{2}\right\|=\bar{\sigma}\left(\Delta A_{2}\right) \leq r_{2} .
$$

The dimension of the uncertainty space is $\ell=n_{1} n_{2}+$ $n_{3} n_{4}$ and it is natural to partition $R^{\ell}$ into its component $x^{1} \in \mathbf{R}^{n_{1} n_{2}}$ associated with the entries of $A_{1}$ and the second component $x^{2} \in \mathbf{R}^{n_{3} n_{4}}$ associated with the entries of $A_{2}$. In view of the above, we now provide a generalization of the framework of Sections 2 and 3 for an arbitrary number of uncertain groups.

5.1 Partitions: $A$ set of positive integers $\mathbf{p}=$ $\left\{\ell_{1}, \ell_{2}, \ldots, \ell_{n}\right\}$ is said to define a partition of $\mathbf{R}^{\ell}$ if $\sum_{i=1}^{n} \ell_{i}=\ell$. Hence, any $x \in \mathbf{R}^{\ell}$ can be represented as a concatenation of $n$ components: $x \doteq\left(x^{1}, x^{2}, \ldots, x^{n}\right)$, where $x^{i} \in \mathbf{R}^{\ell_{i}}$ for $i=1, \ldots n$.

5.2 Unirectangularity: Associated with any partition $\mathbf{p}=\left\{\ell_{1}, \ell_{2}, \ldots, \ell_{n}\right\}$ of $\mathbf{R}^{\ell}$ and a point $x \in \mathbf{R}^{\ell}$, is the rectangular projection set

$$
\begin{array}{r}
\mathcal{R}_{\mathrm{p}}(x) \doteq\left\{y=\left(y^{1}, y^{2}, \ldots, y^{n}\right): y^{i}=\alpha_{i} x^{i}\right. \text { for some } \\
\left.\alpha_{i} \in[0,1], x^{i} \in \mathbf{R}^{\ell_{i}} \text { and } i=1,2, \ldots, n\right\} .
\end{array}
$$

Note that the extreme cases $\mathbf{p}=\{1,1, \ldots, 1\}$ and $\mathbf{p}=$ $\{\ell\}$ correspond to the theory in [5] and [7] respectively.

The rectangular projection set defined above is an $\boldsymbol{n}$-dimensional hyper-rectangle in $\mathbf{R}^{\ell}$ with the segment $[0, x]$ being its principal diagonal. To illustrate, consider the case $\ell=3$ and the minimal partition $p=\{3\}$. Then, for each $x \in \mathbf{R}^{3}$, the set $\mathcal{R}_{\mathbf{p}}(x)$ is just the principal diagonal itself, a one-dimensional segment $[0, x]$; see Figure 6 . On the other extreme, the maximal partition $\mathrm{p}=\{1,1,1\}$ leads to $\mathcal{R}_{\mathrm{p}}(x)$ being the whole three-dimensional rectangle. A nontrivial partition, say $p=\{2,1\}$, corresponds to the two-dimensional rectangle which is represented by the shaded region. 


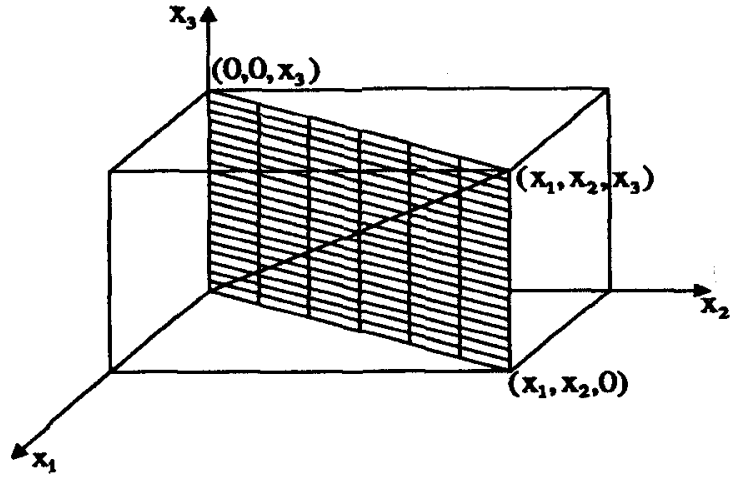

Figure 6: $\mathcal{R}_{\mathrm{p}}(x)$ for Different Partitions

5.3 Generalized Unirectangularity Definition: Given a partition $\mathbf{p}=\left\{\ell_{1}, \ell_{2}, \ldots, \ell_{n}\right\}$ of $\mathbf{R}^{\ell}$, a set $\mathcal{X} \subseteq \mathbf{R}^{\ell}$ is said to be unirectangular, with respect to $\mathrm{p}$ if $\mathcal{R}_{\mathrm{p}}(x) \subseteq \mathcal{X}$ for all $x \in \mathcal{X}$. According to this definition, unirectangularity is a partition-dependent property of a set. To see this, note that the shaded region in Figure 6 is unirectangular with respect to $p_{1}=\{2,1\}$ but it is not unirectangular with respect to $p_{2}=\{1,2\}$ and $p=\{1,1,1\}$. On the other hand, unirectangularity with respect to a partition implies unirectangularity with respect to all coarser partitions.

5.4 Class of Density Functions $\mathcal{F}_{r, p}:$ A partition $p=\left\{\ell_{1}, \ell_{2}, \ldots, \ell_{n}\right\}$ is fixed and the random vector $X=\left(X^{1}, X^{2}, \ldots, X^{n}\right) \in \mathbf{R}^{\ell}$ is obtained with $X^{i} \in$ $\mathbf{R}^{\ell_{i}}$ for $i=1,2, \ldots, n$. It is assumed that the components $X^{i}$ are independent and each component satisfies the assumptions of the setting presented in [7] with support set of the form

$$
Q_{r, i} \doteq\left\{x \in \mathbf{R}^{\ell_{i}}:\|x\| \leq r\right\} .
$$

Namely, a density function $f_{i}$ for $X^{i}$ is admissible if it is radially non-increasing and constant on the boundary of the set $Q_{r, i}$ for all $r \geq 0$. More precisely, $f_{i}$ has the following properties

$$
\begin{aligned}
& \|x\| \leq\|y\| \Rightarrow f_{i}(x) \geq f_{i}(y) ; \\
& \|x\|=\|y\| \Rightarrow f_{i}(x)=f_{i}(y) .
\end{aligned}
$$

Since the components of the partition are independent, the density function of the random vector $X$ is given by $f\left(x^{1}, x^{2}, \ldots, x^{n}\right)=f_{1}\left(x^{1}\right) f_{2}\left(x^{2}\right) \cdots f_{n}\left(x^{n}\right)$. The class of densities satisfying conditions presented in this section is denoted by $\mathcal{F}_{r, p}$ and $X^{f}$ is taken to be a random vector with density $f$. Finally, the uniform distribution over the support set is denoted by $u_{r}$. The generalization of Theorem 2.5 does not include its proof which is quite similar to that given in Section 2 and requiring Theorem 6.3 in [7].

5.5 Theorem: Let $\mathcal{X} \subseteq \mathbf{R}^{\ell}$ be a unirectangular set with respect to $\mathbf{p}$. Then, given $r>0$,

$$
\inf _{f \in \mathcal{F}_{r, p}} \operatorname{Prob}\left\{X^{f} \in \mathcal{X}\right\}=\operatorname{Prob}\left\{X^{u_{r}} \in \mathcal{X}\right\} .
$$

\section{Conclusion}

The results in this paper suggest one obvious direction for further work. Namely, it would be desirable to obtain a sharp estimator $R_{0}(\varepsilon)$ which avoids execution of the algorithm $\mathcal{A}$ for each sample $q^{k}$. Instead, it would be desirable to have a result which makes it possible to estimate $R_{0}(\varepsilon)$ while only testing whether Property $\mathcal{P}$ is satisfied for each sample $q^{k}$; i.e., replace each execution of $\mathcal{A}$ with a $\mathcal{P}$-test. This improvement would greatly facilitate numerical computation.

\section{References}

[1] Stengel, R. F. and L. R. Ray (1991). "Stochastic Robustness of Linear Time-Invariant Systems," IEEE Transactions on Automatic Control, AC-36, pp. 82-87.

[2] Ray, L. R. and R. F. Stengel (1993). "A Monte Carlo Approach to the Analysis of Control Systems Robustness," Automatica, vol. 3, pp. 229-236.

[3] Tempo, R. and E. W. Bai (1996). "Robustness Analysis with Nonlinear Parametric Uncertainty: A Probabilistic Approach," Proceedings of the IEEE Conference on Decision and Control, pp. 3418-3423, Kobe, Japan.

[4] Khargonekar, P. and A. Tikku (1996). "Randomized Algorithms for Robust Control Analysis and Synthesis Have Polynomial Complexity," Proceedings of the IEEE Conference on Decision and Control, pp. 3470-3475, Kobe, Japan.

[5] Barmish, B. R. and C. M. Lagoa (1996). "The Uniformity Principle: A New Tool for Probabilistic Robustness Analysis," Proceedings of the Conference on Information Science and Systems, pp. 929-933, Princeton, 1996; journal version in press for Mathematics of Control, Signals and Systems, 1997.

[6] Barmish, B. R., C. M. Lagoa and P. S. Shcherbakov (1996). "Probabilistic Enhancement of Robustness Margins Provided by Linear Matrix Inequalities," Proceedings of the Allerton Conference on Communication, Control and Computing, pp. 160-169, Monticello, 1996.

[7] Barmish, B. R., C. M. Lagoa and R. Tempo (1996). "Radially Truncated Uniform Distribution for Probabilistic Robustness of Control Systems," Proceedings of American Control Conference, Albuquerque, 1997.

[8] Tsypkin, Ya. Z. and B. T. Polyak (1991). "Frequency Domain Approach to Robust Stability of Continuous Systems," in: Systems and Control: Topics in Theory and Applications (F. Kozin and T. Ono, eds.), pp. 389-399, MITA Press, Osaka. 